\title{
Role of grapsid crabs, Parasesarma erythrodactyla, in entry of mangrove leaves into an estuarine food web: a mesocosm study
}

Caroline Nerot - Tarik Meziane •

Anais Provost-Govrich · Hervé Rybarczyk •

Shing Yip Lee

Published online: 26 August 2009

(C) Springer-Verlag 2009

Erratum to: Mar Biol

DOI 10.1007/s00227-009-1262-6

Unfortunately, the last author's name has been wrongly published. Instead of S. Yip Lee it should be Shing Yip Lee.

The online version of the original article can be found under doi:10.1007/s00227-009-1262-6.

C. Nerot $\cdot$ T. Meziane $(\bowtie) \cdot$ A. Provost-Govrich ·

H. Rybarczyk

UMR BOREA, Muséum National d'Histoire Naturelle,

CNRS 7208, IRD 207, UPMC, 61 Rue Buffon,

75231 Paris Cedex, France

e-mail: meziane@mnhn.fr

C. Nerot · A. Provost-Govrich · S. Y. Lee

Australian Rivers Institute-Coast and Estuaries,

Griffith University, Gold Coast, QLD 4222, Australia 